\title{
·SOLVING THE RATIONAL POLYNOMIAL COEFFICIENTS BASED ON L CURVE
}

\author{
Guoqing Zhou ${ }^{1}$, Xiaozhu Li ${ }^{1}$, Tao Yue ${ }^{1, *}$, Wei Huang ${ }^{1,2}$, Chaoshuang $\mathrm{He}^{1}$, Yu Huang ${ }^{1}$ \\ ${ }^{1}$ Guangxi Key Laboratory of Spatial Information and Geomatics, Guilin University of Technology, No. 12 Jian'gan Road, Guilin, \\ Guangxi, 541004, China- (gzhou, yuetao)@glut.edu.cn \\ ${ }^{2}$ Department of Mechanical and Control Engineering, Guilin University of Technology, No. 12 Jian'gan Road, Guilin, Guangxi \\ 541004, China-102016477@glut.edu.cn
}

\section{Commission III, ICWG III/IVb}

KEY WORDS: RPC model, Tikhonov Regularization method, L Curve, orthorectification, rigorous sensor model, ill conditioned equation

\begin{abstract}
:
The rational polynomial coefficients (RPC) model is a generalized sensor model, which can achieve high approximation accuracy. And it is widely used in the field of photogrammetry and remote sensing. Least square method is usually used to determine the optimal parameter solution of the rational function model. However the distribution of control points is not uniform or the model is over-parameterized, which leads to the singularity of the coefficient matrix of the normal equation. So the normal equation becomes ill conditioned equation. The obtained solutions are extremely unstable and even wrong. The Tikhonov regularization can effectively improve and solve the ill conditioned equation. In this paper, we calculate pathological equations by regularization method, and determine the regularization parameters by $\mathrm{L}$ curve. The results of the experiments on aerial format photos show that the accuracy of the first-order RPC with the equal denominators has the highest accuracy. The high order RPC model is not necessary in the processing of dealing with frame images, as the RPC model and the projective model are almost the same. The result shows that the first-order RPC model is basically consistent with the strict sensor model of photogrammetry. Orthorectification results both the firstorder RPC model and Camera Model (ERDAS9.2 platform) are similar to each other, and the maximum residuals of X and Y are 0.8174 feet and 0.9272 feet respectively. This result shows that RPC model can be used in the aerial photographic compensation replacement sensor model.
\end{abstract}

\section{INTRODUCTION}

The new generation of commercial high-resolution (up to one meter ground resolution) satellite imagery will open a new era for digital mapping (Zhou G, 2000). At present, the RPC model is widely applied to geometry processing of remote sensing images of high-resolution satellite and has become a sensorindependent generalized geometry processing model that can replace the strict geometry processing model (Zhang G, 2006). RPC model independent of the sensor and platform, with excellent interpolation characteristics, given the appropriate number of control information, you can get a high degree of fitting accuracy (Li Deren, 2006). In this paper, we start with the traditional frame-format image, attempt to solve the aerial RPC parameters, and establish an RPC model that can replace its strict physical imaging model. As with satellite images, in the process of solving RPC of aerial images, the normal equations are prone to pathological conditions. The Tikhonov Regularization can be a good solution to the pathological equation. The key of this regularization method is to determine its effective regularization parameters. The commonly used methods are L curve method, GCV method and empirical formula method. In this paper, L-curve method is used to determine regularization parameters. The procedures of orthorectification for digital terrain model (DTM) based and digital building model (DBM) based orthoimage generation and their mergence for true orthoimage generation are discussed in detail (Zhou G, 2005). As the experimental image is mainly urban area, this article uses DBM instead of DEM. The process of DBM based orthoimage generation only orthorectify the displacement caused by buildings without considering displacement caused by terrain (Zhou G, 2008). For the building area, experiments and error analysis show that the third-order RPC model can replace the rigorous imaging model to complete the orthorectification of images.

\section{RFM}

The RFM model relates the ground coordinate to its corresponding image pixel coordinate using a ratio polynomial. For one image, the following ratio polynomial is defined (OGC, 1999; Zhang G, 2000; Sohn H G, 2003):

$$
\left\{\begin{array}{l}
\mathrm{x}=\frac{P_{1}(X, Y, Z)}{P_{2}(X, Y, Z)} \\
y=\frac{P_{3}(X, Y, Z)}{P_{4}(X, Y, Z)}
\end{array}\right.
$$

Where $(\mathrm{X}, \mathrm{Y}, \mathrm{Z})$ is the regularized control point ground coordinates, and $(\mathrm{x}, \mathrm{y})$ is the regularized image pixel coordinates. The polynomial $P_{i} \quad(\mathrm{i}=1,2,3,4)$ consists of a third degree polynomial containing $\mathrm{X}, \mathrm{Y}, \mathrm{Z}$, and has the same independent form of parameter. As follows:

$$
\begin{aligned}
P_{1}(X, Y, Z)= & a_{1}+a_{2} Y+a_{3} X+a_{4} Z+a_{5} Y X+a_{6} Y Z+a_{7} X Z+a_{8} L^{2}+ \\
& a_{9} X^{2}+a_{10} Z^{2}+a_{11} X Y Z+a_{12} Y^{3}+a_{13} Y X^{2}+a_{14} Y Z^{2}+ \\
& a_{15} Y^{2} X+a_{16} X^{3}+a_{17} X Z^{2}+a_{18} Y^{2} Z+a_{19} X^{2} Z+a_{20} Z^{3}
\end{aligned}
$$

* Corresponding author: Tao Yue, Email: yuetao@glut.edu.cn 


$$
\begin{array}{r}
P_{2}(X, Y, Z)=b_{1}+b_{2} Y+b_{3} X+b_{4} Z+b_{5} Y X+b_{6} Y Z+b_{7} X Z+b_{8} L^{2}+ \\
b_{9} X^{2}+b_{10} Z^{2}+b_{11} X Y Z+b_{12} Y^{3}+b_{13} Y X^{2}+b_{14} Y Z^{2}+ \\
b_{15} Y^{2} X+b_{16} X^{3}+b_{17} X Z^{2}+b_{18} Y^{2} Z+b_{19} X^{2} Z+b_{20} Z^{3} \\
P_{3}(X, Y, Z)=c_{1}+c_{2} Y+c_{3} X+c_{4} Z+c_{5} Y X+c_{6} Y Z+c_{7} X Z+c_{8} L^{2}+ \\
c_{9} X^{2}+c_{10} Z^{2}+c_{11} X Y Z+c_{12} Y^{3}+c_{13} Y X^{2}+c_{14} Y Z^{2}+ \\
c_{15} Y^{2} X+c_{16} X^{3}+c_{17} X Z^{2}+c_{18} Y^{2} Z+c_{19} X^{2} Z+c_{20} Z^{3} \\
P_{4}(X, Y, Z)=d_{1}+d_{2} Y+d_{3} X+d_{4} Z+d_{5} Y X+d_{6} Y Z+d_{7} X Z+d_{8} L^{2}+ \\
d_{9} X^{2}+d_{10} Z^{2}+d_{11} X Y Z+d_{12} Y^{3}+d_{13} Y X^{2}+d_{14} Y Z^{2}+ \\
d_{15} Y^{2} X+d_{16} X^{3}+d_{17} X Z^{2}+d_{18} Y^{2} Z+d_{19} X^{2} Z+d_{20} Z^{3}
\end{array}
$$

Where $\mathrm{a}_{i}, \mathrm{~b}_{i}, \mathrm{c}_{i}, \mathrm{~d}_{i}(\mathrm{i}=1, \ldots, 20)$ are the coefficients of $P_{i}(\mathrm{i}=1,2,3,4)$ respectively. Where $\mathrm{b}_{1}=1$ and $\mathrm{d}_{1}=1$.

In the existing RPC model, the optical projection system product rational multiplying for the production error for the first place indication; the errors of earth curvature, atmospheric refraction and lens distortion can be modeled with quadratic terms in rational polynomials; some other unknown errors with high order components, such as the camera vibration, are expressed as three terms in a rational polynomial (Zhang G, 2000; Zheng L, 2007). In order to enhance the stability of the parameter solution, the regularized to between -1 and 1 . Before running a linear algorithm to calculate parameters, it is important to normalize the coordinate data using scale factors and offsets.

$$
\left\{\begin{array}{l}
X_{\mathrm{i}}^{R}=\frac{X_{i}-X_{O}}{X_{S}} \\
X_{O}=\frac{\sum_{i=1}^{n} X_{\mathrm{i}}}{n} \\
X_{S}=\max \left(\left|X_{\max }-X_{O}\right|,\left|X_{\min }-X_{O}\right|\right)
\end{array}\right.
$$

Where $x_{i}$ is the original coordinate, $X_{i}^{R}$ (i $=1,2$, to $\mathrm{n}$ ) is the normalized coordinate.

\section{SOLVING RATIONAL FUNCTION MODEL PARAMETERS BY LEAST SQUARE ESTIMATION}

Equation (1) is rewritten as follows and linearized.

$$
\left\{\begin{array}{l}
F_{x}=P_{1}(X, Y, Z)-x P_{2}(X, Y, Z) \\
F_{y}=P_{3}(X, Y, Z)-y P_{4}(X, Y, Z)
\end{array}\right.
$$

This has the advantage that the coefficient matrix B of the error equation to be avoided contains the parameters to be solved. Without initial value iteration, solve the RPC parameter directly. The error equation can be expressed as follows:

$$
\mathrm{V}=\mathrm{BX}-\mathrm{L}
$$

Among them:

$$
\begin{gathered}
B=\left[\begin{array}{llll}
\frac{\partial F_{x}}{\partial a_{i}} & \frac{\partial F_{x}}{\partial b_{i}} & \frac{\partial F_{x}}{\partial c_{i}} & \frac{\partial F_{x}}{\partial d_{i}} \\
\frac{\partial F_{y}}{\partial a_{i}} & \frac{\partial F_{y}}{\partial b_{i}} & \frac{\partial F_{y}}{\partial c_{i}} & \frac{\partial F_{y}}{\partial d_{i}}
\end{array}\right] \\
X=\left[\begin{array}{llll}
a_{i} & b_{i} & c_{i} & d_{i}
\end{array}\right]^{T}
\end{gathered}
$$

$$
L=\left[\begin{array}{ll}
\mathrm{x} & y
\end{array}\right]^{T}
$$

Least square estimation theory, so that the minimum $V^{T} V$, available the normal equation.

\section{DETECTION OF MORBID EQUATION}

In actual calculation, the inverse matrix of the design matrix is required. When the matrix is ill-formed or rank deficient, the inverse matrix is unstable. Its slight variation has a great influence on the solution result and can not be correctly solved. Before solving the normal equations, we need to detect the extent of morbid equation which is composed by design matrix. Commonly detection methods include the method of condition number, eigenvalue method and determinant method so on.

Determinant method is based on the determinant of matrix equation to determine the ill-posed. When the design matrix determinant tends to zero, the solution of $\mathrm{X}$ satisfies the least square criterion. However, the error itself is very large, the least-squares estimation method fails. When $\operatorname{det}\left(B^{T} B\right)=0$, the solution of $\mathrm{X}$ is not unique, so that the least squares adjustment does not converge.

\section{L CURVE AND REGULARIZATION METHOD}

\subsection{Tikhonov Regularization}

Tikhonov regularization can be a good solution to the pathological equation. The Tikhonov regularization method transforms ill-posed problems into approximate and appropriate problems. The approximate solutions of the original problem are found. In essence, we can find the best approximation solution from them.

According to Tikhonov regularization theory, the regularization function is as follows:

$$
F_{X}=\|B X-L\|^{2}+\|\Gamma X\|^{2}
$$

Where $\Gamma$ is a Tikhonov matrix and usually $\Gamma=\alpha \mathrm{I} . \mathrm{F}_{\mathrm{X}}$ take the minimum, then $X=\left(B^{T} B+\mathrm{k} I\right)^{-1} B^{T} \mathrm{~L}$. Where $\mathrm{k}$ is the regularization parameters and $\mathrm{k}=\alpha^{2}$.

The Tikhonov regularization method is equivalent to the approximate deformation of the least square method. The coefficient matrix is increased by $\mathrm{kI}$, which transforms the original pathological equation into a benign equation approximating the nearest approximation. Finally, we get the stable solution of the parameter. Obviously, the idea of the Tikhonov regularization method to solve this kind of pathological problem can be seen as adding a damping term to the main diagonal of the coefficient matrix of the normal equation (Hu Zhigang, 2010).

\subsection{L curve}

The $\mathrm{L}$ curve method to determine the regularization parameter was first proposed by Hansen P C (Hansen P C, 1991). The singular values of the coefficient matrix $\mathrm{B}$ of the error equation were analyzed to determine the parametric function of $\mathrm{L}$ curve. Due to magnitude differences, it is common to logarithm the two parametric functions.

L curve function is: 


$$
\left\{\begin{array}{l}
\mu(k)=\lg \left\|B X_{\mathrm{k}}-L\right\| \\
v(k)=\lg \left\|X_{k}\right\|
\end{array}\right.
$$

Let $\mu(k)$ be the horizontal axis and the vertical axis $\nu(k)$ be the $\mathrm{L}$ curve. The regularization parameter $\mathrm{k}$ is the maximum curvature of the L curve.

According to the curve derivation formula:

$$
k=\frac{\mu^{\prime}(k) v^{\prime \prime}(k)-\mu(k)^{\prime \prime} v(k)}{\left(\mu^{\prime}(k)^{2}+v(k)^{2}\right)^{3 / 2}}
$$

\section{SOLVE THE RPC EXPERIMENT AND THE PRECISION ANALYSIS}

A pair of WILD-RC30 aerial image is used as experimental data The resolution is 0.3 feet. The impact area is 5054 feet $X$ 5054feet. It is located in downtown Denver, USA, mainly including middle and high-rise buildings (Figure 1). See Table 1 and Table 2 for more information on the impact.

The rigorous imaging model (collinearity equation) of the image is as follows (Kim H, 2004):

$$
\left\{\begin{array}{l}
x^{\prime}-x_{0}=-f \frac{a_{1}\left(X-X_{S}\right)+b_{1}\left(Y-Y_{S}\right)+c_{1}\left(Z-Z_{S}\right)}{a_{3}\left(X-X_{S}\right)+b_{3}\left(Y-Y_{S}\right)+c_{3}\left(Z-Z_{S}\right)} \\
y^{\prime}-y_{0}=-f \frac{a_{2}\left(X-X_{S}\right)+b_{2}\left(Y-Y_{S}\right)+c_{2}\left(Z-Z_{S}\right)}{a_{3}\left(X-X_{S}\right)+b_{3}\left(Y-Y_{S}\right)+c_{3}\left(Z-Z_{S}\right)}
\end{array}\right.
$$

\begin{tabular}{|c|c|}
\hline Camera Name & RC30_5236_13248_92297 \\
\hline Calibrated Focal Length & $153.022 \mathrm{~mm}$ \\
\hline Scale & $1: 7200$ \\
\hline Image Size & $17054 \times 17054$ \\
\hline
\end{tabular}

Table 1. Image basic information

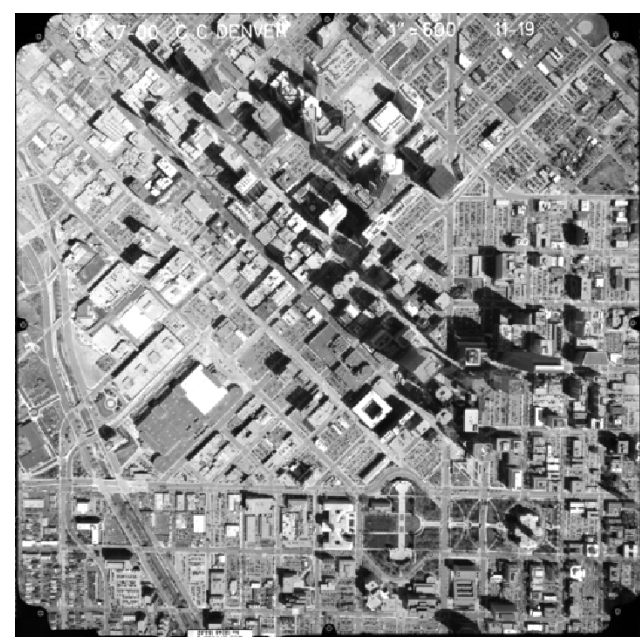

Figure 1. Original image

\subsection{Independent of terrain method}

There are two main solutions to RPCs: terrain-related programs and terrain-independent programs.

6.1.1. Terrain-related programs: In the case of unknown model parameters, we can not determine the precise correspondence between the control points and the corresponding pixel coordinates. The pixel coordinates corresponding to the control points are measured by the features of the geomorphic geomorphology, and the error of the coordinates of the image points is larger than that of the strict model. And the number of control points that can be collected is limited. Therefore, when the sensor model is too complicated to build, or the precision is not very high, this method is is adopted.

6.1.2. Independent of the terrain program: First we establish a three-dimensional control grid uniform control points. Then, the pixel coordinates corresponding to the control point are obtained from the rigorous imaging model to solve the RPC parameters as known conditions.

\begin{tabular}{|c|c|}
\hline elements & data \\
\hline $\mathrm{f}$ & $153.022 \mathrm{~mm}$ \\
\hline $\mathrm{x}_{o}$ & $0.002 \mathrm{~mm}$ \\
\hline $\mathrm{y}_{o}$ & $-0.004 \mathrm{~mm}$ \\
\hline$X_{S}$ & $3143040.487824465 \mathrm{ft}$ \\
\hline$Y_{S}$ & $1696520.187562254 \mathrm{ft}$ \\
\hline$Z_{S}$ & $9073.690373855898 \mathrm{ft}$ \\
\hline$\varphi$ & 1.705248003481724 \\
\hline$\omega$ & 0.08706879816433863 \\
\hline$K$ & 89.02474746577455 \\
\hline
\end{tabular}

Table 2. Internal and external orientation elements

\begin{tabular}{|c|c|c|c|}
\hline \multirow{2}{*}{ Order } & Denominator & RFC number & Min GCP \\
\cline { 2 - 4 } & $P_{2}=\mathrm{P}_{4}$ & 11 & 4 \\
\hline \multirow{2}{*}{2} & $P_{2} \neq \mathrm{P}_{4}$ & 14 & 7 \\
\cline { 2 - 4 } & $P_{2}=\mathrm{P}_{4}$ & 29 & 15 \\
\hline \multirow{2}{*}{3} & $P_{2} \neq \mathrm{P}_{4}$ & 38 & 19 \\
\cline { 2 - 4 } & $P_{2}=\mathrm{P}_{4}$ & 59 & 30 \\
\hline
\end{tabular}

Table 3. Six kinds of RPC

Step:

1. The scope of the stereoscopic grid is determined according to the geographical coordinates of the 4 points of the image, and the maximum and minimum elevation values of the DEM in the area corresponding to the area. The control grid of the $\mathrm{i}$ row of 
the $\mathrm{j}$ column $\mathrm{k}$ layer is set up, and the grid size is recorded $\mathrm{i} \times$ $\mathrm{j} \times \mathrm{k}$.

2. From result of positive calculation of the control point, the pixel coordinates are obtained. The control grid points are brought into the collinear equation, and the coordinates of the image point are calculated, and then the pixel coordinates are obtained.

3. The control points and pixels need to be normalized before participating in the calculation.

4. Solve RPC parameters.

5. Error calculation and accuracy analysis. After finding the RPC parameters, it is necessary to test its accuracy and feasibility. Like control grid, we set up check grid. The pixel coordinates of the check point are calculated by solving the RPC model and the rigorous imaging model respectively, and the accuracy and feasibility of the RPC model are measured by using the differences of the pixel coordinates calculated by the two models.

\subsection{Experiments and Results}

Specifically, six forms of RPCs can be combined (see Table 3) based on the difference and order of the denominators of the rational function model (1st order, 2 nd order, and 3rd order).

6.2.1. Test: Independent of the terrain program: A control grid with a size of $20 \times 20$ and a height of 5 levels determines 4000 control points. And a check grid with a size of $10 \times 10$ and a height 5 levels were established for testing and analysis. In order to evaluate the fitting accuracy of RPC model, the pixel coordinates of each checkpoint were calculated by strict sensor model and RPC model, and the error is calculated on the basis of the difference between the two. The results are shown in Table 4 .

\begin{tabular}{|c|c|c|c|}
\hline order & denominator & $\mathrm{x}$ & $\mathrm{y}$ \\
\hline \multirow{2}{*}{$1^{\text {st }}$ order } & $P_{2} \neq \mathrm{P}_{4}$ & $2.6616 \mathrm{e}-10$ & $3.0926 \mathrm{e}-10$ \\
\cline { 2 - 4 } & $P_{2}=\mathrm{P}_{4}$ & $1.4096 \mathrm{e}-10$ & $1.3465 \mathrm{e}-10$ \\
\hline \multirow{2}{*}{$2^{\text {nd }}$ order } & $P_{2} \neq \mathrm{P}_{4}$ & $4.3410 \mathrm{e}-10$ & $4.8376 \mathrm{e}-10$ \\
\cline { 2 - 4 } & $P_{2}=\mathrm{P}_{4}$ & $2.3897 \mathrm{e}-10$ & $2.0551 \mathrm{e}-10$ \\
\hline \multirow{2}{*}{$3^{\text {rd }}$ order } & $P_{2} \neq \mathrm{P}_{4}$ & $5.9436 \mathrm{e}-09$ & $8.7761 \mathrm{e}-9$ \\
\cline { 2 - 4 } & $P_{2}=\mathrm{P}_{4}$ & $5.9840 \mathrm{e}-09$ & $8.6601 \mathrm{e}-9$ \\
\hline
\end{tabular}

Table 4. Maximum errors (pixels) in image for the aerial photograph data

According to the experiment, the following results can be obtained. When the order is first order and second order, the accuracy of RPC with the equal denominators is significantly higher than that with unequal denominator. And regardless of whether the denominators are the same or not, the accuracy of the first-order RPC is higher than that of the second order and the third order. More specifically, it is clear that the accuracy of the first-order RPC with the equal denominators has the highest accuracy. This result shows that RFM can be used in the aerial photographic compensation replacement sensor model (Tao, C. V.).

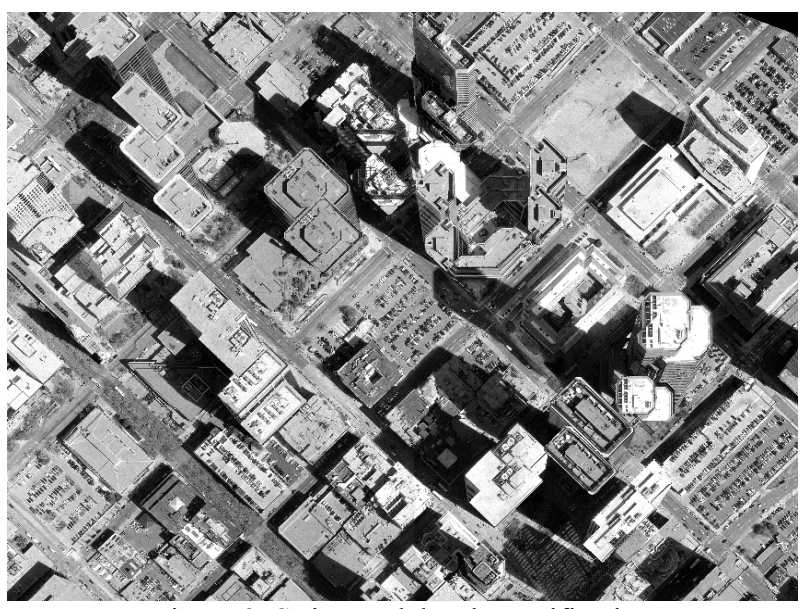

Figure 2. Strict model orthorectification

According to the error analysis, it is clear that the third-order RPC has the highest accuracy. There is no significant difference between the two cases of denominator equality and denominator. Due to the limited range of DEM, only the orthorectification results of some image regions can be obtained. Figure 1 was respectively orthorectified using a rigorous imaging model and a first-order RPC model (different denominators).

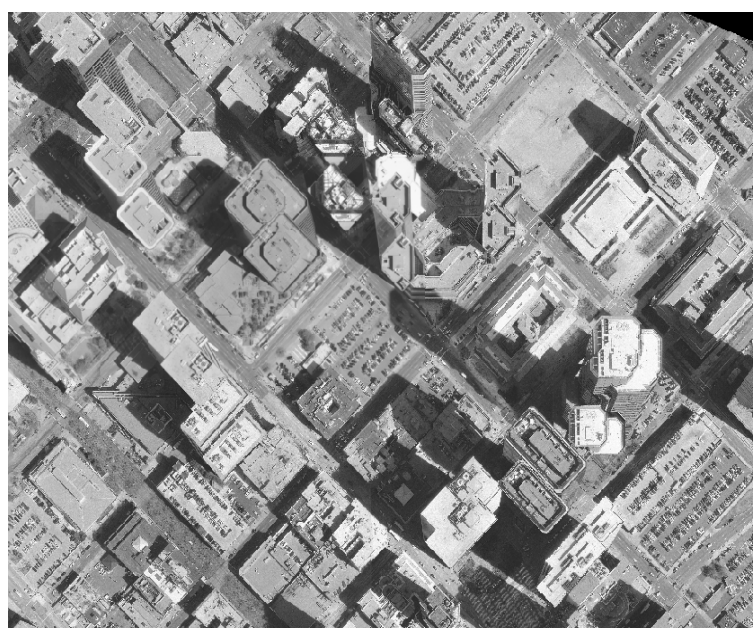

Figure 3. Third order RPC model orthorectification

The results are shown in Figure 2, Figure 3. Orthorectification results of the two models are basically the same, again shows that the RPC model can be completed instead of strict imaging model orthorectification, and the maximum residuals of $\mathrm{X}$ and $\mathrm{Y}$ are 0.8174 feet and 0.9272 feet respectively.

\section{CONCLUSION}

In this paper, RPCs are solved directly without initial value. This method is simple and convenient. As the least square method fails, the Tikhonov regularization method is used to solve the ill-conditioned problem. The regularization parameters are determined by the L curve. Set independent of the terrain experimental program. Control points and check points are generated by the $3 \mathrm{D}$ space grid. High-order RPC model is not necessary in the processing of dealing with frame images, as the RPC model and the projective model are almost the same. This result shows that the first-order RPC model is 
basically consistent with the strict sensor model of photogrammetry. Orthorectification results both the first-order RPC model and Camera model are similar to each other. This result shows that RPC model can be used in the aerial photographic compensation replacement sensor model.

\section{ACKNOWLEDGEMENTS}

This paper is financially supported by the National Natural Science of China under Grant numbers 41431179, The National Key Research and Development Program of China under Grant numbers 2016YFB0502500, the State Oceanic Administration under Grant numbers 2014\#58, GuangXi Natural Science Foundation under grant numbers 2015GXNSFDA139032, and 2012GXNSFCB0530, Guangxi Science \& Technology Development Program under the Contract numbers GuiKeHe 14123001-4 and GuangXi Key Laboratory of Spatial Information and Geomatics Program under Grant numbers 151400701,151400712 , and 163802512 .

\section{REFERENCES}

Hansen, P. C., \& O'Leary, D. P. (1991). The use of the L-curve in the regularization of discrete II-posed problems. University of Maryland at College Park.

Hu Zhigang, Hua Xianghong. (2010). Determining Tikhonov Regularization Parameters Using Optimal Regularization Method. Science and Technology of Surveying and Mapping, 35 (2): 51-53.

Kim, H., \& Lee, J. (2004). RPC model generation from the physical sensor model. Remote Sensing (Vol.5234, pp.94239434). International Society for Optics and Photonics.

Li Deren, Zhang G, Jiang Wanshou, et al. (2006). Regional network adjustment for RPC model of SPOT-5 HRS images lacking control points. Journal of Wuhan University (Information Science Edition), 31 (5): 377-381.

OGC (OpenGIS Consortium), (1999). The OpenGIS Abstract Specification-Topic 7: The Earth Imagery Case. URL: http://www.opengis.org/public/abstract/99-107.pdf.

Sohn, H. G., Park, C. H., \& Yu, H. U. (2003). Application of rational function model to satellite images with the correlation analysis. Ksce Journal of Civil Engineering, 7(5), 585-593.

Tao, C. V., \& Hu, Y. (2001). A comprehensive study of the rational function model for photogrammetric processing. Photogrammetric Engineering \& Remote Sensing, 67(12), 1347-1357.

Zheng L, Chen Yingying, Lin Yi. (2007). RPC Correction based on SVD algorithm. [J] .Surveying and Mapping Engineering, 2007, 16: 30-32.

Zhang G. (2005). High resolution satellite remote sensing image geometric correction without control points. Wuhan University.

Zhang G, Yuan X. (2006). On RPC Model of Satellite Imagery. Journal of Geospatial Information Science (English version), 9(4):285-292.
Zhou, G. (2000). Accuracy evaluation of ground points from ikonos high-resolution satellite imagery. Photogrammetric Engineering \& Remote Sensing, 66(9), 1103-1112.

Zhou, G., Chen, W., Kelmelis, J. A., \& Zhang, D. (2005). A comprehensive study on urban true orthorectification. IEEE Transactions on Geoscience \& Remote Sensing, 43(9), 21382147.

Zhou, G., \& Chen, W. (2008). Urban image true orthorectification in the national map program. 